Pacific Journal of Mathematics

SOME RESULTS ON PRÜFER RINGS 


\title{
SOME RESULTS ON PRÜFER RINGS
}

\author{
Thomas G. LuCAS
}

For a domain $D, D$ is a Prüfer domain if and only if $D / P$ is a Prüfer domain for every prime ideal $P$ of $D$. The same result does not hold for rings with zero divisors. In this paper it is shown that for a Prüfer ring $R$ with prime ideal $P, R / P$ is a Prüfer ring if $P$ is not properly contained in an ideal consisting entirely of zero divisors. An example is provided to show that, in general, this is the best possible result. According to M. Boisen and P. Sheldon, a pre-Prüfer ring is defined to be a ring for which every proper homomorphic image is a Prüfer ring. In this paper it is proved that for a pre-Prüfer ring $R$ containing zero divisors, the integral closure of $R$ is a Prüfer ring. Furthermore, if $R$ is a reduced pre-Prüfer ring with more than two minimal prime ideals, then $R$ is already integrally closed and, moreover, $R$ is not only Prüfer but arithmetical as well. An example is provided of an integrally closed pre-Prüfer domain which is not a Prüfer domain.

1. Introduction. In this paper, all rings are assumed to be commutative with nonzero unit. A regular element of a ring $R$ is one which is not a divisor of zero, and a regular ideal is one which contains a regular element. By an overring we mean a ring between $R$ and $T(R)$, the total quotient ring of $R$. All unexplained terminology is standard as in [5] and [8].

For commutative rings with zero divisors, M. Griffin [6] defined a Prüfer ring as a ring for which every finitely generated regular ideal is invertible. He showed that with suitable modifications many of the properties which characterize Prüfer domains also characterize Prüfer rings; some of these are collected in Proposition 2.1 of this paper. One purpose of this paper is to examine what can be said about the relation between a ring $R$ being Prüfer and $R / P$ being a Prüfer domain for each prime ideal $P$ of $R$. It is not the case that the two conditions are equivalent. In fact, in general neither implies the other. In $\$ 2$ we provide the following positive results. First, if $P$ is either a regular prime ideal or maximal with respect to containing only zero divisors, then $R / P$ is a Prüfer ring if $R$ is a Prüfer ring (Proposition 2.2). In the event that $T(R)$ is von Neumann regular, then every prime ideal of $R$ satisfies the above condition. Thus if $R$ is Prüfer and $T(R)$ is von Neumann regular, then $R / P$ is a Prüfer domain for every prime ideal $P$ of $R$. Moreover, if $R / P$ 
is a Prüfer domain for every prime ideal $P$ of $R$ and $T(R)$ is von Neumann regular, then the integral closure of $R$ is a Prüfer ring (Proposition 2.6).

A ring $R$ is said to be arithmetical if the ideals of $R_{M}$ are linearly ordered for every maximal ideal $M$ of $R$. Arithmetical rings are always Prüfer rings, but not conversely. However, if $R$ is Prüfer and $T(R)$ is von Neumann regular, then $R$ is also arithmetical [6; Theorems 19 and 20]. In our Proposition 2.6, we give a new characterization of reduced arithmetical rings; namely, a reduced ring $R$ is arithmetical if and only if $R / P$ is a Prüfer domain for every prime ideal $P$ of $R$ and for every pair of minimal prime ideals $P$ and $Q, R / P \cap Q$ is integrally closed.

In $\$ 3$ we determine when rings formed by the technique of idealization of a module are Prüfer rings. For a ring $R$ and $R$-module $B$, the idealization of $B$ is the ring $R(+) B$ formed from the direct sum $R \oplus B$ by defining multiplication of elements $(r, m)$ and $(s, n)$ of $R(+) B$ by

$$
(r, m) \cdot(s, n)=(r s, r n+s m) .
$$

Using idealization it is possible to transform any given ring $R$ into a Prüfer ring of the form $R(+) B$. In particular, if $B=\sum R / M_{\alpha}$ where the $M_{\alpha}$ range over the maximal ideals of $R$, then $R(+) B$ is its own total quotient ring and, hence, is trivally a Prüfer ring. Our Proposition 3.1 characterizes those rings of the form $R(+) B$ which are Prüfer rings. In particular, if $R$ is a Prüfer ring and $B$ is any $R$-module, then the integral closure of $R(+) B$ is a Prüfer ring. In Example 3.3 we present a Prüfer ring $R(+) B$ where $R$ is not a Prüfer ring nor is $R(+) B$ a total quotient ring.

2. Prüfer and pre-Prüfer rings. One of the many characteristics of Prüfer domains is that a domain $D$ is Prüfer if and only if $D / P$ is a Prüfer domain for every prime ideal $P$ of $D$. This same result does not hold for rings with zero divisors. In particular, there exists a ring $R$ for which every proper homomorphic image of $R$ is a Prüfer ring yet $R$ is not a Prüfer ring [3; Example 3.1]. However, if $R$ is a reduced ring such that $R / P$ is a Prüfer domain for every prime ideal $P$ of $R$ and $R / P \cap Q$ is integrally closed for every pair of prime ideals $P$ and $Q$, then $R$ is not only Prüfer but is in fact arithmetical (Proposition 2.6). Also, if $T(R)$ is von Neumann regular and $R / P$ is a Prüfer domain for every prime ideal $P$ of $R$, then the integral closure of $R$ is Prüfer. On the other hand, if $R$ is a Prüfer ring, then $R / P$ need not be a Prüfer domain (Example 3.6); though it will be if $P$ is either regular or maximal with respect to containing only zero divisors (Proposition 2.2). While this latter result can 
be derived as a corollary to a proposition due to $M$. Boisen and $\mathbf{P}$. Sheldon [3; Proposition 2.3] we shall provide a proof which roughly parallels the proof given in [5] for Prüfer domains.

Before presenting this proof, we state without proof the following proposition which is a standard result about Prüfer rings.

PROPOSITION 2.1. The following are equivalent for a ring $R$.

(a) $R$ is a Prüfer ring.

(b) If $A B=A C$ where $A, B, C$ are ideals of $R$ with $A$ finitely generated and regular, then $B=C$.

(c) $R$ is integrally closed and for any $a, b \in R$ at least one of which is regular, $(a, b)^{2}=\left(a^{2}, b^{2}\right)$.

(d) For each maximal ideal $M$ of $R$, if $A$ and $B$ are ideals of $R$ with $A$ regular, then either $A R_{M} \subset B R_{M}$ or $B R_{M} \subset A R_{M}$.

(e) Every overring of $R$ is integrally closed.

Proposition 2.2. Let $R$ be a Prüfer ring and $P$ be a prime ideal of $R$. If $P$ is either regular or maximal with respect to containing only zero divisors, then $R / P$ is a Prüfer domain.

Proof. If $P$ is a maximal ideal, there is nothing to prove. Hence, we assume that every ideal properly containing $P$ is regular and let $M$ be an arbitrary but fixed maximal ideal properly containing $P$. We will show that $(R / P)_{M / P}$ is a valuation domain.

To this end, let $A$ and $B$ be ideals, necessarily regular, which properly contain $P$ and are contained in $M$. It is enough to show that $(A / P)_{M / P}$ and $(B / P)_{M / P}$ are comparable.

As $A$ and $B$ are regular ideals of a Prüfer ring, either $A R_{M} \subset B R_{M}$ or $B R_{M} \subset A R_{M}$. Hence, since $(R / P)_{M / P}$ and $R_{M} / P R_{M}$ are canonically isomorphic, either $(A / P)_{M / P} \subset(B / P)_{M / P}$ or $(B / P)_{M / P} \subset(A / P)_{M / P}$. Since $M, A$, and $B$ were arbitrary, the ideals of $(R / P)_{M / P}$ are linearly ordered for every maximal ideal $M$ containing $P$ and thus $R / P$ is a Prüfer domain.

In §3, we give an example to show that the above result is the best possible without further assumptions.

Our next result concerns rings which contain nonzero nilpotent elements.

Proposition 2.3. Let $R$ be a nonreduced ring with nilradical $N$. If $R / N$ is a Prüfer ring, then a necessary and sufficient condition for $R$ to be Prüfer is for $R$ to be integrally closed.

Proof. Assume that $R / N$ is a Prüfer ring. As all Prüfer rings are integrally closed, the necessity is apparent. 
Assume that $R$ is integrally closed. To establish the sufficiency of this assumption, we make use of Proposition 2.1(c).

Let $a, b \in R$ with at least one of these a regular element, say $b$. As $R / N$ is a Prüfer ring, and $\bar{b}$ is a regular in $R / N,(\bar{a}, \bar{b})^{2}=\left(\bar{a}^{2}, \bar{b}^{2}\right)$. Thus there exist $r, s \in R$ such that $a b=r a^{2}+s b^{2}+n$ for some $n \in N$. But as $R$ is integrally closed and $b$ is regular, $b N=b^{2} N=N$. Hence for some $m \in N, n=m b^{2}$ and $a b=r a^{2}+(s+m) b^{2}$. Therefore $(a, b)^{2}=\left(a^{2}, b^{2}\right)$ and $R$ is a Prüfer ring.

If $R$ is a reduced ring such that $T(R)$ is von Neumann regular, then every prime ideal of $R$ is either regular or maximal with respect to containing only zero divisors since only the minimal primes contain only zero divisors. Thus if $R$ is also a Prüfer ring, then $R / P$ is a Prüfer domain for every prime ideal $P$ of $R$. The converse is nearly true and the following example not only shows that the converse fails but also suggests how to obtain a slightly modified version of the converse.

EXAMPLE 2.4. (cf. [3; Example 3.1]). Let $R=K[X, Y] /(X Y)$ where $K$ is a field. Then the only nonmaximal primes are $P=(X) /(X Y)$ and $Q=(Y) /(X Y)$. As $R / P \cong K[Y]$ and $R / Q \cong K[X]$ are Prüfer domains, we have that $R / M$ is a Prüfer domain for every prime ideal $M$ of $R$. Since $R$ is not integrally closed, it cannot be a Prüfer ring. However, the integral closure of $R$ is isomorphic to $K[X] \oplus K[Y]$ which is a Prüfer ring.

The above example generalizes to any reduced ring with exactly two minimal prime ideals as the following lemma shows. The lemma proves useful in our proof of Proposition 2.6.

LEMMA 2.5. Let $R$ be a reduced ring with exactly two minimal prime ideals $P$ and $Q$. If both $R / P$ and $R / Q$ are Prüfer domains, then the integral closure of $R$ is arithmetical and hence is a Prüfer ring.

Proof. Since $R$ is reduced, $P \cap Q=(0)$ and thus $R$ can be embedded in $R / P \oplus R / Q$. Moreover, $R / P \oplus R / Q$ is integral over $R$ since both $(1,0)$ and $(0,1)$ satisfy the polynomial equation $X^{2}-X=0$. Thus if both $R / P$ and $R / Q$ are Prüfer domains, then the integral closure of $R$ in $T(R / P \oplus R / Q)$ is exactly $R / P \oplus R / Q$ and hence is arithmetical. As $T(R)$ is isomorphic to $T(R / P \oplus R / Q$ ), the integral closure of $R$ (in $T(R))$ is arithmetical.

In a manner similar to that used in Example 2.4, we can construct a ring $R$ with any finite number of minimal prime ideals $P_{1}, \ldots, P_{n}$, $n>2$, such that $R / P_{i}$ is a Prüfer domain for each $i$, but $R$ is not 
Prüfer. For example for $n=3$, let $R=K\left[X_{1}, X_{2}, X_{3}\right] / I$ where $I=$ $\left(X_{1} X_{2}, X_{1} X_{3}, X_{2} X_{3}\right)$. Then the minimal primes of $R$ are $P_{1}=\left(X_{2}, X_{3}\right) / I$, $P_{2}=\left(X_{1}, X_{3}\right) / I$, and $P_{3}=\left(X_{1}, X_{2}\right) / I$. Thus $R / P_{i} \cong K\left[X_{i}\right]$ is a Prüfer domain, but as in the example $R$ is not integrally closed. Of note is the fact that for $i \neq j, R / P_{i} \cap P_{j} \cong K\left[X_{i}, X_{j}\right] /\left(X_{i} X_{j}\right)$ is not integrally closed.

Our next two results deal with those reduced rings $R$ for which $R / P$ is a Prüfer domain for every prime ideal $P$ of $R$. In the first of these, Proposition 2.6., we use the above lemma to show that if in addition to $R / P$ being Prüfer for every prime ideal $P, R / P \cap Q$ is integrally closed for every pair of minimal prime ideals $P$ and $Q$, then $R$ is not only Prüfer but arithmetical as well. The converse also holds, for if $R$ is arithmetical, then $R / I$ is arithmetical for every ideal $I$ of $R$.

Proposition 2.6. Let $R$ be a reduced ring such that $R / P$ is a Prüfer domain for every prime ideal $P$ of $R$. If for each pair of minimal prime ideals $P$ and $Q$ of $R, R / P \cap Q$ is integrally closed, then $R$ is arithmetical and hence Prüfer.

Proof. Let $P$ and $Q$ be a pair of distinct minimal prime ideals of $R$ such that $\bar{R}=R / P \cap Q$ is integrally closed. Then $\bar{R}$ has two minimal prime ideals, namely $\bar{P}=P / P \cap Q$, and $\bar{Q}=Q / P \cap Q$. As $\bar{R} / \bar{P} \cong R / P$ and $\bar{R} / \bar{Q} \cong R / Q$ are both Prüfer domains and $\bar{R}$ is integrally closed, we have by the above lemma that $\bar{R}$ is arithmetical. In particular, if $M$ is a maximal ideal of $R$ containing $P \cap Q$, then $M$ must contain exactly one of $P$ and $Q$ since $\bar{M}=M / P \cap Q$ is such that $\bar{R}_{\bar{M}}$ is a valuation domain.

If $R / P \cap Q$ is integrally closed for every pair of minimal prime ideals $P$ and $Q$, then it must be that no maximal ideal contains more than one minimal prime. Hence for each maximal ideal $M$ there is a unique minimal prime ideal $P$ contained in $M$, in which case $R_{M}=(R / P)_{M / P}$ is a valuation domain. Therefore $R$ is arithmetical.

In [4; Propositions 5 and 6], S. Endo proved that a reduced ring with von Neumann regular total quotient ring is integrally closed if and only if it is locally an integrally closed domain. Using this result we consider the case of a reduced ring $R$ for which $T(R)$ is von Neumann regular and $R / P$ is a Prüfer domain for each prime ideal $P$ of $R$.

Proposition 2.7. Let $R$ be a reduced ring such that $T(R)$ is von Neumann regular. If $R / P$ is a Prüfer domain for every prime ideal $P$ of $R$, then the integral closure of $R$ is a Prüfer ring.

Proof. Assume that $R / P$ is a Prüfer domain for every prime ideal $P$ of $R$. Let $R^{\prime}$ be the integral closure of $R$. then for each maximal ideal $M$ of $R^{\prime}, R_{M}^{\prime}$ is a domain. We will show that $R_{M}^{\prime}$ is a valuation domain. To 
this end, let $M$ be a fixed but arbitrary maximal ideal of $R^{\prime}$. Since $R_{M}^{\prime}$ is a domain, there is a unique minimal prime ideal $Q$ contained in $M$ and $R_{M}^{\prime}=\left(R^{\prime} / Q\right)_{M / 2}$. Let $P=R \cap Q$, then $R / P$ is naturally embedded in $R^{\prime} / Q$ and viewed in this way the two rings have the same quotient field. Hence, since $R / P$ is Prüfer, so is $R^{\prime} / Q$. Therefore, $R_{M}$ is a valuation domain and $R^{\prime}$ is Prüfer.

In [3], Boisen and Sheldon define a pre-Prüfer ring as a ring $R$ for which every proper homomorphic image of $R$ is a Prüfer ring. In other words, $R$ is a pre-Prüfer ring if for every proper ideal $I$ of $R, R / I$ is a Prüfer ring. It turns out that Prüfer domains are integrally closed pre-Prüfer domain [2; Proposition 1], but not conversely (Example 2.8). For rings with zero divisors, Prüfer rings are not necessarily pre-Prüfer rings. Examples abound for this conclusion, including [3; Example 3.3]. However, if $R$ is a pre-Prüfer ring where $Z(R) \neq(0)$, then the integral closure of $R$ is a Prüfer ring. The proof of this statement for reduced rings relies heavily on Lemma 2.5. For nonreduced rings we use the fact that if $N$ and $N^{\prime}$ are the respective nilradicals of $R$ and $R^{\prime}$, then $R / N \subset R^{\prime} / N^{\prime} \subset$ $T(R / N)$.

PROPOSITION 2.8. Let $R$ be a pre-Prüfer ring which is not a domain.

(1) If $R$ is reduced and has three or more minimal prime ideals, then $R$ is arithmetical.

(2) If $R$ is reduced with exactly two minimal prime ideals, then the integral closure of $R$ is arthmetical.

(3) If $R$ is nonreduced, then the integral closure of $R$ is a Prüfer ring.

Proof. If $R$ is reduced and has three or more minimal prime ideals, then $P \cap Q \neq(0)$ for any pair of minimal primes $P$ and $Q$. Hence $R / P \cap Q$ is a Prüfer ring for any two minimal primes $P$ and $Q$. Thus $R$ is arithmetical by Proposition 2.6.

Statement (2) is simply a restatement of Lemma 2.5.

Statement (3) follows from Proposition 2.3 and the remark above concerning $R / N$ and $R^{\prime} / N^{\prime}$.

Our first example shows how to make an integrally closed pre-Prüfer domain which is not Prüfer from an arbitrary Prüfer domain using a $D+M$ construction.

EXAmple 2.9. Let $D$ be a Prüfer domain with quotient field $k$. For indeterminates $X$ and $Y$, let $K=k(Y)$ and let $V$ be the valuation domain $K+X K[[X]]$. Then $V$ is one dimensional with maximal ideal $M=$ $X K[[X]]$. The ring $R=D+M$ is an integrally closed pre-Prüfer domain which is not Prüfer. 
As the quotient field of $D$ is not $K, R$ is not Prüfer. However, $R$ is integrally closed since $D$ is integrally closed in $K$. (These and subsequent results concerning $D+M$ constructions can be found in [5]).

To see that $R$ is a pre-Prüfer domain, let $I$ be a nonzero ideal of $R$. As $I$ compares with $M$, we have two cases to consider: either $I$ contains $M$, in which case $I=J+M$ for some ideal $J$ of $D$, or $I$ is properly contained in $M$. In the first case, $R / I \cong D / J$ which is a Prüfer ring [2; Proposition 2]. On the other hand, if $I$ is properly contained in $M$, then $R / I$ is a nonreduced ring with nilradical $M / I$. Hence, if $A$ is a finitely generated regular ideal of $R / I$, then $A=(J+M) / I$ where $J$ is a finitely generated (invertible) ideal of $D$. As $J+M=J R, J+M$ is invertible in $R$, and thus $A$ is invertible [3; Proposition 2.3]. Therefore, $R$ is a pre-Prufer domain.

Proposition 2.10. Let $R$ be a ring such that $R_{M}$ is a Prüfer ring for every maximal ideal $M$ of $R$. Then $R$ is a Prüfer ring.

Proof. Let $A, B, C$ be ideals of $R$ such that $A B=A C$ and $A$ is finitely generated and regular. By Proposition 2.1(b), it is enough to show that this implies $B=C$. We will show that $B R_{M}=C R_{M}$ for every maximal ideal $M$ of $R$ and from this conclude that $B=C$. To this end let $M$ be an arbitrary but fixed maximal ideal of $R$. Then $A R_{M}$ is still a finitely generated regular ideal and $A B R_{M}=A C R_{M}$. Since $R_{M}$ is a Prüfer ring, $B R_{M}=C R_{M}$. Hence $B=C$ and $R$ is a Prüfer ring.

Our next example shows that a Prüfer ring is not necessarily locally a Prüfer ring.

EXAMPLE 2.11. (cf. [7]). Let $D=K[X, Y]$ where $K$ is an algebraically closed field and $X$ and $Y$ are indeterminates. Let $\mathscr{A}$ be an index set for the set of maximal ideals of $D$ and let $K=\mathscr{A} \times N$ where $N$ is the set of natural numbers. For each $i=(\alpha, n) \in I$, let $M_{i}=M_{\alpha}$ and $k_{i}=D / M_{i}$. Let $S=\prod_{i \in I} K_{i}$ and $B=\sum_{i \in I} K_{i}$. Finally, let $R=A+B$ where $A$ is the image of $D$ in $S$ under the canonical homomorphism $f$ defined by $f(d)_{i}=d+M_{i}$.

It is straightforward to check that $R$ is a total quotient ring with maximal ideals $P$ of two forms: either $P=P_{\alpha}=f\left(M_{\alpha}\right)+B$ or $P=P_{i}=$ $\left\{r \in R ; r_{i}=0\right\}$. In the second case, $P_{i}$ is also a minimal prime and $R_{P_{i}} \cong k_{i}$. However, in the first case $R_{P_{\alpha}} \cong D_{M_{\alpha}}$ since $B$ is the minimal prime ideal contained in $P$. As $D_{M_{\alpha}}$ is not a Prüfer domain, $R$ is not locally Prüfer.

3. Idealization. Given any ring $R$, it is possible to find an $R$-module $B$ such that the idealized ring $R(+) B$ is Prüfer. For example, let $B=$ $\sum R / M_{\alpha}$ where the $M_{\alpha}$ range over the maximal ideal of $R$. Then $R(+) B$ 
is Prüfer since it is its own total quotient ring. However, if we ask for $R(+) B$ to be a Prüfer ring which is not a total quotient ring, there may exist no module such that $R(+) B$ is a Prüfer ring. For example, if $R$ is a Noetherian ring with no invertible maximal ideals, then $R(+) B$ is Prüfer if and only if it is a total quotient ring. On the other hand, if $R$ is a Prüfer ring which is not a total quotient ring, then such a module always exists. In particular, $R(+) T(R)$ is a Prüfer ring which is not a total quotient ring. However, our Example 3.3 shows that $R$ need not be Prüfer before there exists a module $B$ such that $R(+) B$ is a nontrivial Prüfer ring. Before presenting this example, we give a characterization of Prüfer rings of the form $R(+) B$.

Proposition 3.1. Let $R$ be $a$ ring and $B$ an $R$-module. Let $Z(B)=$ $\{r \in R ; r b=0$ for some nonzero $b \in B\}$ and let $S=R \backslash[Z(R) \cup Z(B)]$.

(a) If $S$ contains only units of $R$, then $R(+) B$ is a total quotient ring and hence Prüfer.

(b) If $S$ properly contains the units of $R$, then the following are equivalent:

(i) $R(+) B$ is Prüfer;

(ii) $B=B_{S}$ and every finitely generated ideal $I$ of $R$ with $I \cap S \neq \varnothing$ is invertible as an ideal of $R$.

Proof. It is elementary to check an element $(r, b)$ of $R(+) B$ is regular if and only if $r \in S$. Moreover, $(r, b)$ is a unit if and only if $r$ is a unit of $R$. The proof of (a) is now apparent.

To prove the equivalence of (i) and (ii), first note that the total quotient ring of $R(+) B$ can be identified with $R_{S}(+) B_{S}$. Hence, if $R(+) B$ is Prüfer, then $B=B_{S}$ for otherwise $R(+) B$ cannot be integrally closed. Also if $B=B_{S}$ and $I$ is an ideal of $R$, then $I(+) B$ is an ideal of $R(+) B$ with inverse $(I(+) B)^{-1}=\left(I^{-1} \cap R_{S}\right)(+) B$. Moreover, in the case that $I \cap S \neq \varnothing$, each $r \in I^{-1}$ is of the form $r=$ $t s^{-1}$ for some $t \in R$ and $s \in I \cap S$. Hence, $I^{-1} \subset R_{S}$ and $(I(+) B)^{-1}=$ $I^{-1}(+) B$.

Assume that $R(+) B$ is a Prüfer ring and let $I$ be a finitely generated ideal of $R$ with $I \cap S \neq \varnothing$. The ideal $I(+) B$ is a regular ideal of $R(+) B$ since $I \cap S \neq \varnothing$. Moreover, if $\left\{a_{1}, \ldots, a_{n}\right\}$ generate $I$ as an ideal of $R$, then $\left\{\left(a_{1}, 0\right), \ldots,\left(a_{n}, 0\right)\right\}$ generate $I(+) B$ as an ideal of $R(+) B$, since $s^{-1} b \in B$ for all $b \in B$ and each $s \in I \cap S$. Hence, $I(+) B$ is a finitely generated regular ideal of $R(+) B$. Thus $I(+) B$ is invertible with inverse $I^{-1}(+) B$. Whence $I I^{-1}=R$ and $I$ is invertible as an ideal of $R$.Conversely assume that $B=B_{S}$ and every finitely generated 
ideal $I$ of $R$ with $I \cap S \neq \varnothing$ is invertible as an ideal of $R$. Since $B=B_{S}$, every regular ideal of $R(+) B$ is of the form $I(+) B$ for some ideal $I$ of $R$ with $I \cap S \neq \varnothing$.

Let $I(+) B$ be a finitely generated regular ideal of $R(+) B$. Then $I$ is a finitely generated ideal of $R$ such that $I \cap S \neq \varnothing$. Hence, by our hypothesis, $I$ is invertible. As above $I^{-1} \subset R_{S}$ since $I \cap S \neq \varnothing$ (and $S$ contains only regular elements). Furthermore, $(I(+) B)^{-1}=I^{-1}(+) B$ and $(I(+) B)(I(+) B)^{-1}=I I^{-1}(+) B=R(+) B$. Therefore, $R(+) B$ is a Prüfer ring.

COROllary 3.2. If $R$ is a Prüfer ring and $B$ is any $R$-module, then $R$ (+) $B_{S}$ is a Prüfer ring, where $S$ is as in Proposition 3.1.

To construct an example of a Prüfer ring of the form $R(+) B$ which is not a total quotient ring where $R$ is not a Prüfer ring we first make use of a $D+M$ construction for the ring $R$. In particular, let $V=Q[[X, Y]]$ be the ring of formal power series in two variables over the field of rational numbers $Q$. Then for $M=(X, Y), V=Q+M$. Let $D=Z_{(2)}$ and $R=D+M$. Then $M$ is simultaneously the maximal ideal of $V$ and a prime ideal of $R$. Furthermore, $R_{M}=V$ and hence $M R_{M}=M$.

EXAMPLE 3.3. Let $R$ be the ring defined above and let $B=(R / M)_{M}$. Then the ring $R(+) B$ is a Prüfer ring which is not a total quotient ring nor is $R$ a Prüfer ring.

The ideals of $R$ which properly contain $M$ are of the form $I+M=$ $I R$ where $I$ is an ideal of $Z_{(2)}$. But the ideals of $Z_{(2)}$ are all principal ideals of the form $I=\left(2^{n}\right), n \geq 1$. Hence the ideals of $R$ which properly contain $M$ are principal. Since $Z(B)=M, R(+) B$ is a Prüfer ring.

The ring $R$ is not Prüfer since the ideal $(X, Y) R$ is not invertible as an ideal of $R$. Also, $R(+) B$ is not a total quotient ring. For example, the element $(2,0)$ is neither a unit nor a zero divisor of $R(+) B$.

In Proposition 3.5 we generalize the above example. Before doing so, however, we need the notion of a valuation ring.

For a ring $R$, a subring $V$ of $R$ is called a valuation ring of $R$ if there exists a prime ideal $P$ of $V$ such that for each $r \in R \backslash V$ there exists a $p \in P$ such that $r p \in V \backslash P$. The pair $(V, P)$ is called a valuation pair of $R$. In the case that $R=T(V), V$ is simply called a valuation ring and $(V, P)$ a valuation pair [9].

Valuation rings differ from valuation domains in many ways. For example, a valuation ring need not have a unique (regular) maximal ideal nor must it be Prüfer. However, if $(V, P)$ is a valuation pair with $V \neq T(V)$, then $V$ is Prüfer if and only if $P$ is the unique regular maximal ideal [1; Theorem 2.3]. Also, if $V$ is a local Prüfer ring, then it is also a 
valuation ring [6; Theorem 13]. Hence the ring $R(+) B$ in the example above is a valuation ring.

The valuation rings of the form $R(+) B$ are characterized by the following lemma. We will use Lemma 3.4 to generalize Example 3.3.

LEMMA 3.4. Let $R$ be $a$ ring and let $B$ be an $R$-module with $S=$ $R \backslash[Z(R) \cup Z(B)]$. Then the ring $R(+) B$ is a valuation ring if and only if $R$ is a valuation ring of $R_{S}$ and $B=B_{S}$.

Proof. Assume that $R(+) B$ is a valuation ring. Then by definition there exists a prime ideal $Q=P(+) B$ such that for each $(s, b) \in$ $T(R(+) B) \backslash R(+) B$ there exists $(r, a) \in P(+) B$ with $(s, b) \cdot(r, a) \in$ $R(+) B \backslash P(+) B$. As $T(R(+) B)$ can be identified with $R_{S}(+) B_{S}$ and $B=B_{S}$ since $R(+) B$ is integrally closed, we have that for each $s \in R_{S} \backslash R$ there exists $r \in P$ such that $r s \in R \backslash P$. Hence, $(R, P)$ is a valuation pair of $R_{S}$ and $R$ is a valuation ring of $R_{S}$.

The proof of the converse is similar and is omitted.

Proposition 3.5. Let $D$ be a local domain with maximal ideal $M$. Let $P$ be a prime ideal of $D$ such that $P D_{P}=P$. For $B=D / P$, the following are equivalent:

(1) $D(+) B_{P}$ is a Prüfer ring;

(2) $D(+) B_{P}$ is a valuation ring;

(3) $D / P$ is a valuation domain;

(4) For any pair $a, b \in D \backslash P$, either $(a) \subset(b)$ or $(b) \subset(a)$.

Proof. If $P=M$, there is nothing to prove. Hence we assume that $P \neq M$.

Since $D$ is local, so is $D(+) B_{P}$. Thus by remarks preceding the proposition, (1) implies (2).

Let $D(+) B_{P}$ be a valuation ring. To see that (2) implies (1), we first show that $(D, M)$ is a valuation pair of $D_{P}$.

By Lemma 3.4, $D$ is a valuation ring of $D_{P}$. Thus for $r \in D_{P} \backslash D$ there exists an $m \in M$ and an $s \in M \backslash P$ such that $r=m s^{-1}$ since $M$ contains all of the nonunits of $D$. Thus by the fact that $P D_{P}=P$, $s P=P$. Hence, if $m \in P$, then there is an $n \in P$ such that $m=n s$. But then $r=(n s) s^{-1}=n \in D$ which violates the assumption that $r \in D_{P} \backslash D$. Hence $m \in M \backslash P$ and $r^{-1}=s m^{-1} \in D_{P}$. As $D$ is a valuation ring of $D_{P}, r^{-1} \in D$ and hence $r^{-1} \in M$. Thus $(D, M)$ is a valuation pair of $D_{P}$. Whence $\left(D(+) B_{P}, M(+) B_{P}\right)$ is a valuation pair and, by remarks preceding the lemma, $D(+) B_{P}$ is a Prüfer ring.

The scheme for the remainder of the proof is to show that (3) and (4) are equivalent, (4) implies (1), and (1) implies (3).

First we shall show that (3) and (4) are equivalent. 
Since $P D_{P}=P$, if $a, b \notin P$, then $a P=P=b P$. Thus for any pair $a, b \notin P,(a) \subset(b)$ if and only if $(a) / P \subset(b) / P$. But a domain is a valuation domain if and only if the ideals are linearly ordered. The equivalence of (3) and (4) now follows.

To see that (4) implies (1), note that principal ideals which are also regular are always invertible. If (4) holds, then every finitely generated ideal of $D$ which is not contained in $P$, contains $P$ and is principal. Thus every finitely generated regular ideal of $D(+) B_{P}$ is principal and $D(+) B_{P}$ is a Prüfer ring.

The final implication $((1) \Rightarrow(3))$ follows from the fact that the only prime ideal of $D$ maximal with respect to missing the set $S=D \backslash P$ is $P$. Hence, $P(+) B_{P}$ is the only prime ideal maximal with respect to containing only zero divisors. Therefore, by our Proposition 2.2, $\left(D(+) B_{P}\right) /\left(P(+) B_{P}\right)=D / P$ is Prüfer, whence a valuation domain.

Our final example shows that Proposition 2.2 is the best possible.

EXAMPLE 3.6. Let $D$ be a 1-dimensional domain which is not a Prüfer domain. Let $B=\sum D / M_{\alpha}$ where the $M_{\alpha}$ range over the maximal ideals of $D$. Then $D(+) B$ is a total quotient ring, hence a Prüfer ring. The only nonmaximal prime ideal of $D(+) B$ is (0) $(+) B=B$ and $(D(+) B) / B=D$ which is not a Prüfer domain.

\section{REFERENCES}

[1] M. Boissen Jr. and M. Larsen, Prüfer and valuation rings with zero divisors, Pacific J. Math., 40 (1972), 7-12.

[2] __ On Prüfer rings as images of Prüfer domains, Proc. Amer. Math. Soc., 40 (1973), 87-90.

[3] M. Boisen Jr. and P. Sheldon, Pre-Prüfer rings, Pacific J. Math., 58 (1975), 331-344.

[4] S. Endo, On semi-hereditary rings, J. Math. Soc., Japan, 13 (1961), 109-119.

[5] R. Gilmer, Multiplicative Ideal Theory, Marcel Dekker, New York, 1972.

[6] M. Griffin, Prüfer rings with zero divisors, J. Reine Angew Math., 240 (1970), 55-67.

[7] M. Griffin, Valuations and Prüfer rings, Canad. J. Math., 26 (1974), 412-429.

[8] M. Larsen and P. McCarthy, Multiplicative Theory of Ideals, Academic Press, New York, 1971.

[9] M. Manis, Valuations on commutative rings, Proc. Amer. Math. Soc., 20 (1969), 193-198.

Received April 4, 1985. The material in section 3 of this paper is taken from the author's Ph.D. dissertation at the University of Missouri. While researching the material in section 2 , the author was supported in part by funds from the Foundation of the University of North Carolina at Charlotte and the State of North Carolina. 



\section{PACIFIC JOURNAL OF MATHEMATICS EDITORS}

\author{
V. S. VARADARAJAN \\ (Managing Editor) \\ University of California \\ Los Angeles, CA 90024 \\ Herbert Clemens \\ University of Utah \\ Salt Lake City, UT 84112 \\ R. FINN \\ Stanford University \\ Stanford, CA 94305
}

\author{
HERMANN FLASCHKA \\ University of Arizona \\ Tucson, AZ 85721 \\ RAMESH A. GANGOLLI \\ University of Washington \\ Seattle, WA 98195 \\ VAUGHAN F. R. JONES \\ University of California \\ Berkeley, CA 94720 \\ ROBION KIRBY \\ University of California \\ Berkeley, CA 94720
}

C. C. MOORE

University of California

Berkeley, CA 94720

H. SAMELSON

Stanford University

Stanford, CA 94305

HAROLD STARK

University of California, San Diego La Jolla, CA 92093

\section{ASSOCIATE EDITORS}
R. ARENS
E. F. BECKENBACH (1906-1982)
B. H. NEUMANN
F. WOLF
K. YosHIDA

\section{SUPPORTING INSTITUTIONS}

\begin{abstract}
UNIVERSITY OF ARIZONA
UNIVERSITY OF BRITISH COLUMBIA

CALIFORNIA INSTITUTE OF TECHNOLOGY

UNIVERSITY OF CALIFORNIA

MONTANA STATE UNIVERSITY

UNIVERSITY OF NEVADA, RENO

NEW MEXICO STATE UNIVERSITY OREGON STATE UNIVERSITY
\end{abstract}

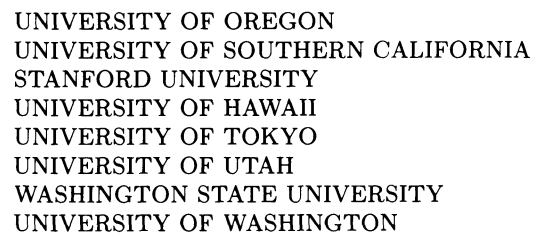

The Supporting Institutions listed above contribute to the cost of publication of this Journal, but they are not owners or publishers and have no responsibility for its content or policies.

Mathematical papers intended for publication in the Pacific Journal of Mathematics should be in typed form or offset-reproduced (not dittoed), double spaced with large margins. Please do not use built up fractions in the text of the manuscript. However, you may use them in the displayed equations. Underline Greek letters in red, German in green, and script in blue. The first paragraph must be capable of being used separately as a synopsis of the entire paper. In particular it should contain no bibliographic references. Please propose a heading for the odd numbered pages of less than 35 characters. Manuscripts, in triplicate, may be sent to any one of the editors. Please classify according to the scheme of Math. Reviews, Index to Vol. 39. Supply name and address of author to whom proofs should be sent. All other communications should be addressed to the managing editor, or Elaine Barth, University of California, Los Angeles, California 90024.

There are page-charges associated with articles appearing in the Pacific Journal of Mathematics. These charges are expected to be paid by the author's University, Government Agency or Company. If the author or authors do not have access to such Institutional support these charges are waived. Single authors will receive 50 free reprints; joint authors will receive a total of 100 free reprints. Additional copies may be obtained at cost in multiples of 50 .

The Pacific Journal of Mathematics is issued monthly as of January 1966. Regular subscription rate: $\$ 190.00$ a year (5 Vols., 10 issues). Special rate: $\$ 95.00$ a year to individual members of supporting institutions.

Subscriptions, orders for numbers issued in the last three calendar years, and changes of address should be sent to Pacific Journal of Mathematics, P.O. Box 969, Carmel Valley, CA 93924, U.S.A. Old back numbers obtainable from Kraus Periodicals Co., Route 100, Millwood, NY 10546.

The Pacific Journal of Mathematics at P.O. Box 969, Carmel Valley, CA 93924 (ISSN 0030-8730) publishes 5 volumes per year. Application to mail at Second-class postage rates is pending at Carmel Valley, California, and additional mailing offices. Postmaster: send address changes to Pacific Journal of Mathematics, P.O. Box 969, Carmel Valley, CA 93924.

PUBLISHED BY PACIFIC JOURNAL OF MATHEMATICS, A NON-PROFIT CORPORATION Copyright (C) 1986 by Pacific Journal of Mathematics 


\section{Pacific Journal of Mathematics}

Vol. 124, No. $2 \quad$ June, 1986

Philip Lee Bowers, Nonshrinkable "cell-like" decompositions of $s \ldots \ldots .257$

Aurelio Carboni and Ross Street, Order ideals in categories .......... 275

Leoni Dalla, Increasing paths on the one-skeleton of a convex compact set in

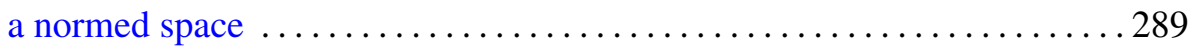

Jim Hoste, A polynomial invariant of knots and links ............... 295

Sheldon Katz, Tangents to a multiple plane curve ................... 321

Thomas George Lucas, Some results on Prüfer rings $\ldots \ldots \ldots \ldots \ldots \ldots 33$

Pham Anh Minh, Modular invariant theory and cohomology algebras of

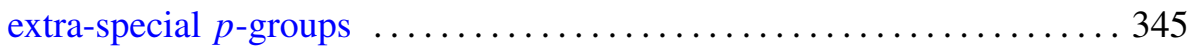

Ikuko Miyamoto, On inclusion relations for absolute Nörlund

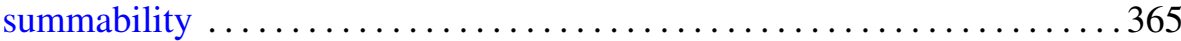

A. Papadopoulos, Geometric intersection functions and Hamiltonian flows on the space of measured foliations on a surface ............. 375

Richard Dean Resco, J. Toby Stafford and Robert Breckenridge

Warfield, Jr., Fully bounded $G$-rings $\ldots \ldots \ldots \ldots \ldots \ldots \ldots \ldots \ldots 4$

Haskell Paul Rosenthal, Functional Hilbertian sums .................417

Luen-Fai Tam, Regularity of capillary surfaces over domains with corners: borderline case ................................. 469

Hugh C. Williams, The spacing of the minima in certain cubic lattices .....483 